\title{
A SATELLITE TEST OF THE EQUIVALENCE PRINCIPLE
}

\author{
R. Reinhard', P.W. Worden ${ }^{2}$ and C.W.F. Everitt ${ }^{2}$
}

\author{
1 Space Science Department of ESA, ESTEC \\ Noordwijk, The Netherlands
}

\author{
2 Stanford University, Stanford \\ California, USA
}

The Satellite Test of the Equivalence Principle (STEP) aims to determine any difference in the rate of fall of test masses in an Earth-orbiting satellite to one part in $10^{17}$ of the total gravitational acceleration - a $10^{6}$ factor improvement over existing measurements. It is being developed by the European Space Agency (ESA) as a collaborative venture with the US National Aeronautics and Space Administration (NASA).

A test of the Equivalence Principle in Earth orbit was proposed as early as 1970 by P.K. Chapman of MIT; the first concept of the STEP experiment was presented by two of the authors (P.W.W. and C.W.F.E.) in 1972 , who then carried out further development for the next 17 years. In November 1989, they, together with European colleagues, proposed the experiment to ESA in response to the Organization's Call for Mission Proposals for the Second Medium-Size Mission (M2) in the context of the long-term Horizon 2000 programme (see page 209). We shall outline the STEP experiment, notably the scientific objectives and implementation of this fundamental physics space mission.

\section{The Equivalence Principle}

STEP is the modern version of an experiment attributed to Galileo which involved dropping two weights from the leaning Tower of Pisa. It compares the inertial to

Rüdeger Reinhard received his Ph.D in physics from the University of Kiel, Germany, in 1976 and then joined ESA's Space Science Department at ESTEC. He moved from solar particle research to become Project Scientist for Giotto, coordinator of the Solar-Terrestial Science Project and finally the Study Scientist for STEP.

Paul W. Worden is a Senior Research Associate with the W.W. Hansen Experimental Physics Lab, Stanford University, Stanford, CA 94305, USA. He studied at Rice University and Stanford, receiving his Ph.D in 1976 for work on testing the equivalence principle.

Francis Everitt studied physics at Imperial College, London, specialising in plate tectonics. He moved to the University of Pennsylvania in 1960 to work on liquid helium, and later to Stanford University. He is a Professor in Stanford's W.W. Hansen Lab and Principle Investigator on NASA's Gravity Probe B and has written extensively on the history of physics. gravitational mass ratios of the test masses, any difference appearing as a difference in the rate of fall. One of the most mysterious facts or near-facts of physics is that this ratio turns out to be with very high precision the same for all bodies, regardless of composition.

This apparent identify between the two kinds of mass profoundly puzzled Newton. Einstein raised it to the level of a postulate - the Equivalence Principle - and generalised it to say that all of the non-gravitational laws of physics (i.e., the laws of special relativity) are the same in any local, freely-falling, non-rotating reference frame. He made this postulate the basis of his new theory of gravitation replacing Newton's theory, known as General Relativity. It follows that any violation of the uniqueness of free fall also violates the Einstein Equivalence Principle, and hence Einstein's Theory of General Relativity.

Newton, using pendulums, determined the Equivalence Principle to be correct to one part in $10^{3}$. Using a torsion balance, Eötvös, in 1896, achieved a sensitivity of $5 \times 10^{-8}$. Since then others, using progressively more refined experiments, have achieved a sensitivity of about $10^{-11}$.

Experiments of the ground are limited for different reasons depending on the type of measurement: free-fall techniques by the time and distance of fall, and torsion balance experiments by the small driving acceleration and the seismic noise background.

Going into space offers the possibility of improving the accuracy by a factor of $10^{6}$. To achieve this very high accuracy, the test masses would be placed in a nearcircular orbit around the Earth, radius $R_{E^{\prime}}$ at several hundred kilometres in altitude. In this way, the test masses never strike the ground, and any difference in the rate of fall can build up for a long time. The experiment is roughly equivalent to a free fall from a tower $R_{E} / \pi \mathrm{km}$ in height, with the difference that the signal is periodic and the experiment can be repeated several thousand times.

\section{STEP}

\section{Scientific significance}

Einstein's Theory of General Relativity, developed in 1915, is recognised as one of the most brilliant of all intellectual achievements. In his Special Theory of Relativity (1905), Einstein had successfully reconciled Maxwell's electromagnetic theory of light with the rest of physics, and then understood by modifying the underlying assumptions of Newton's mechanics. Having done this, he was confronted with a new problem - how to interpret gravity. According to special relativity, no signal can propagate faster than light, but according to Newton's theory of gravitation, gravity is a force acting instantaneously at a distance. Einstein's eventual answer was that gravity is not a force in that sense but a field acting through space, somewhat resembling the Maxwellian electromagnetic field but operating at a deeper level, modifying the very structure of space and time.

Although splendidly successful, Einstein's theory has in turn at least three deep problems which suggest that it will have to be amended:

1) As Einstein himself eventually reluctantly admitted, the beautiful underlying idea of geometrising all of physics only partly works. Einstein struggled for 40 years to extend this idea into a modified theory of gravitation and electricity but failed.

2) Equally disturbing, the strenuous efforts of many physicists to reconcile general relativity and quantum mechanics have also failed.

3) More generally, progress that has been taking place in parts of physics, especially in the rather successful unification between electromagnetic forces and the weak interaction underlying $\beta$-decay, seems to suggest that the way forward to a more global verification such as Einstein hoped for may be of a different kind than Einstein had supposed. In particular, there are some fairly broadly-based reasons for expecting that the next step forward in gravitational theory may be a theory that is at some level non-metric, i.e., one in which the gravitational interaction cannot be entirely reduced to a process of distorting time-space.

If this conjecture is true then the Equivalence Principle will certainly be violated experimentally at some level. The lines of theoretical evidence are far from clear or 
certain, but there are reasons for thinking that violations may occur at around the $10^{-15}$ level. Such considerations make STEP especially exciting: seldom does one have an opportunity in physics to make an advance of a factor $10^{6}$ in measuring a quantity as fundamental or as mysterious as equivalence.

\section{Experiment}

The core of the STEP experiment consists of three accelerometers each containing two test masses (Fig. 1a): superconducting magnetic bearings constrain the test masses to one-dimensional motion (the $x$-direction). Each accelerometer measures any differential acceleration of its two test masses. The high accuracy of $10^{-12} \mathrm{~mm}$ in detecting differential displacements is achieved using SQUID magnetometers.

Five different materials will be used for the $\approx 1 \mathrm{~kg}$ test masses (tentatively hydrogen as polyethylene, copper, niobium, beryllium, and gold). Two test masses should be of the same material to see if area-proportional forces (e.g., residual gas pressure, electrostatic charging) are a significant disturbance.

The accelerometers are accommodated in a cryogenic dewar (Fig. 1b) containing roughly 200 I of superfluid helium which will last about six months. Boil-off will be used as a propellant for the spacecraft's so-called drag-free thrusters activated in such a way as to force the spacecraft to follow the motion of a proof mass (one of the test masses - see below).

The main disturbances inside the dewar stem from gas pressure and temperature effects. The internal gas pressure and the temperature difference across the experiment must be as small as possible to achieve the desired accuracy $\left(10^{-13}\right.$ torr and $10^{-3} \mathrm{~K}$ can be realised by cooling to $2 \mathrm{~K}$ to freeze residual gases and to take advantage of the large heat conductivity of superfluid helium). Very low temperatures are also required to operate the SQUID's, to reduce the thermal expansion of the apparatus, and to provide nearly perfect electromagnetic shielding using superconducting lead shields.

\section{Co-Experiments \\ Constant of gravity}

The STEP spacecraft provides an extraodinarily quiet "laboratory", giving rise to the possibility of measuring the fundamental gravitation constant $G$ very precisely. The constant is presently only known to about one part in $10^{3}$ so the masses of all celestial bodies are known only to that accuracy. If one mass in an outer accelerometer is displaced, the spacecraft compensates for the gravitational attraction between it and the other mass by selfaccelerating (rotating). This rotation can be observed by the spacecraft's star-trackers with arcsecond accuracy, corresponding to one part in $10^{5}$ in the measure-
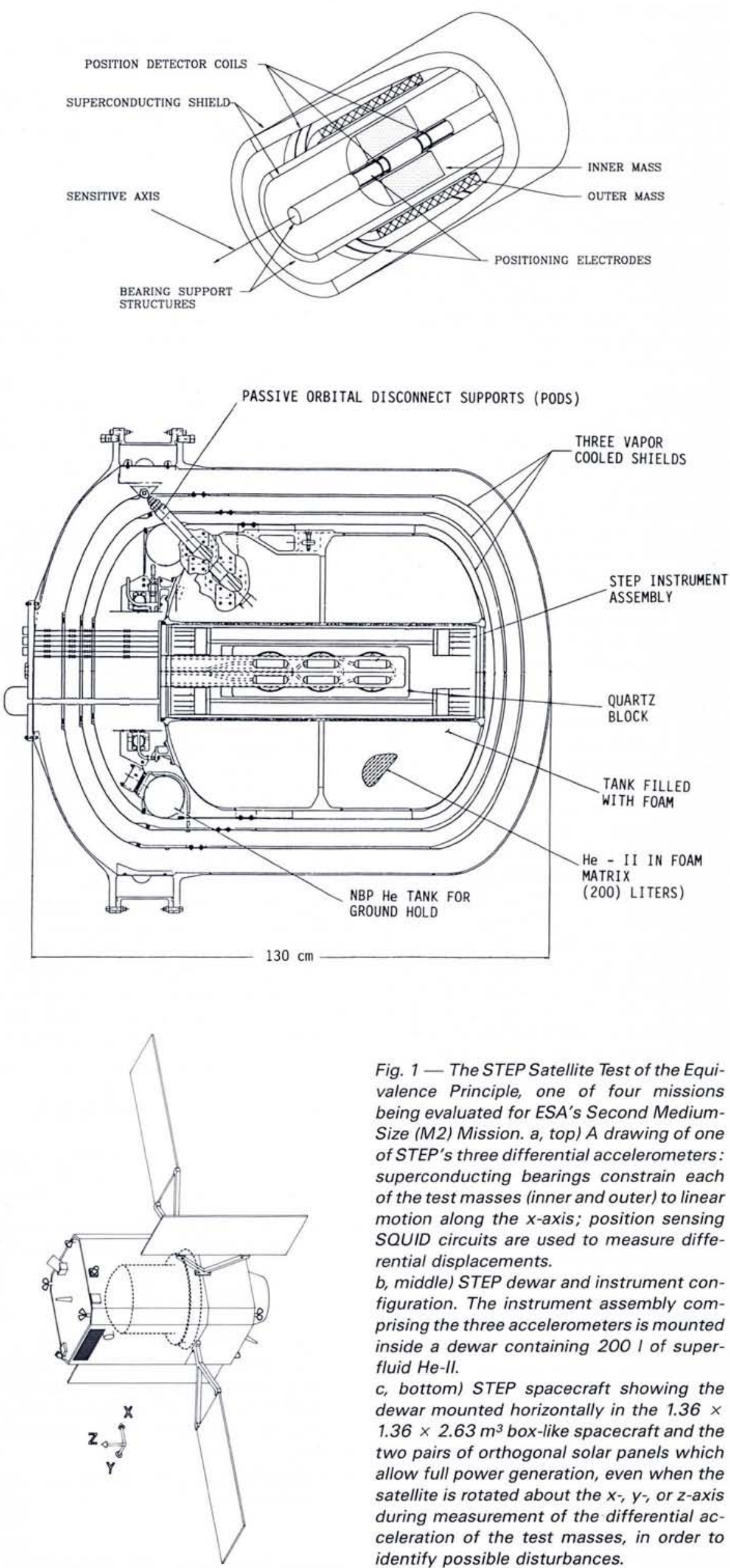

Fig. 1 - The STEP Satellite Test of the Equivalence Principle, one of four missions being evaluated for ESA's Second MediumSize (M2) Mission. a, top) A drawing of one of STEP's three differential accelerometers: superconducting bearings constrain each of the test masses (inner and outer) to linear motion along the $x$-axis; position sensing SQUID circuits are used to measure differential displacements.

$b$, middle) STEP dewar and instrument configuration. The instrument assembly comprising the three accelerometers is mounted inside a dewar containing 200 I of superfluid $\mathrm{He}-\mathrm{Il}$.

$c$, bottom) STEP spacecraft showing the dewar mounted horizontally in the $1.36 x$ $1.36 \times 2.63 \mathrm{~m}^{3}$ box-like spacecraft and the two pairs of orthogonal solar panels which allow full power generation, even when the satellite is rotated about the $x$ - $y$-, or $z$-axis during measurement of the differential acceleration of the test masses, in order to identify possible disturbances. 
ment of $G$, essentially a 100 -fold improvement (Fig. 2).

\section{Geodesy}

Tracking a drag-free satellite with high precision (of the order of a few tens of $\mathrm{mm}$ ) allows improvement of data describing the Earth's gravity field (geopotential), in particular of the lower harmonics. STEP offers a geodesy experiment of remarkable interest as it combines in a single satellite a relatively low altitude $(550 \mathrm{~km})$ with drag-free and gradiometer measurements. The proposal is to track the STEP spacecraft using the Global Positioning System (GPS) in a differential mode and by laser ranging.

\section{Aeronomy}

The trust data from STEP's drag-free controller will provide valuable information to the aeronomy community. The thrust keeping the spacecraft in a pure gravitational orbit is proportional to the drag force, which in turn depends on the atmospheric density, temperature, motion, and composition. STEP can provide data with excellent time resolution which would be especially valuable for the verification and further improvement of thermospheric dynamic models of the Earth.

\section{Spacecraft}

The dewar containing the STEP experiment is centrally mounted inside the three-axis stabilised STEP spacecraft (Fig. 1c) having the shape of a rectangular box (with the edges cut off to fit inside the launch vehicle). The spacecraft measures $1.36 \times 1.36 \times 2.63 \mathrm{~m}^{3}$ and weighs 824 $\mathrm{kg}$ at launch of which $226 \mathrm{~kg}$ is payload. Power is provides by $2 \mathrm{~m}^{2}$ solar panels mounted such that full power is available when the spacecraft is in its normal position and also rotated $90^{\circ}$ about the $y$-axis.

An essential function of the STEP satellite is to "shield" the test masses from any environmental disturbance (mostly air drag). The test masses are completely surrounded by a shield which follows a proof mass (one of the test masses) without touching it. This is done by measuring the position of the proof mass and compensating for any displacement by firing a combination of the 16 proportional thrusters. STEP will be drag-free to $7 \times$ $10^{-12} \mathrm{~g} / \sqrt{ } \mathrm{Hz}$, this being the power spectral density of accelerations which can be expected to average to $<10^{-14} \mathrm{~mm} / \mathrm{s}^{2}$ in an observation time of one week.

Attitude measurements with an accuracy of a few arcseconds are provided by three star-tracker systems. Scientific, spacecraft and experimental housekeeping data are transmitted at a rate of $\mathbf{4 2 . 6}$ kbits/s and commands can be up-linked at $2 \mathrm{kbit} / \mathrm{s}$. Data can be stored on-board in a $14 \mathrm{Mbit}$ solid state memory during periods with no contact with the ground.

Launch, orbit analysis and operations

The STEP spacecraft will be launched

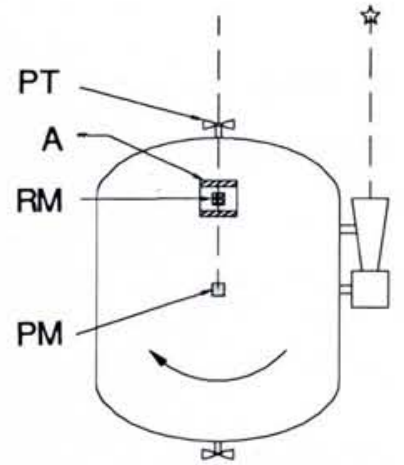

DRAG FREE ROTATION Reference mass keeps

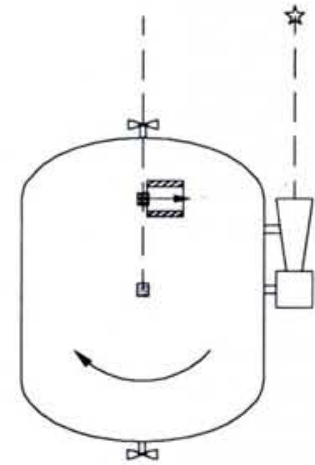

SELF-ACCELERATION IN ANGLE Gravity of attractor

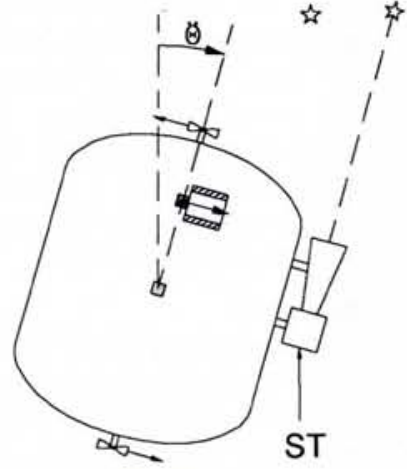

MEASUREMENT OF G

Self-acceleration 8 measured by star tracker

Fig. 2 - Determining the gravitational constant to high accuracy - one of STEP's coexperiments. During drag-free orbiting (left) with one of the central accelerometer's masses as the proof mass, the mass in one of the two outer accelerometers is displaced (middle) and the spacecraft compensates by rotating: it is repositioned by the firing of proportional thrusters controlled by a star-tracker (right). PT - proportional thrusters; A - attractor; $R M$ - reference mass; PM - proof mass; ST - star-tracker.

by a commercially available four-stage Taurus rocket from the Western Test Range in California, USA, and placed in a Sun-synchronous (near polar) orbit with a $550 \mathrm{~km}$ perigee and a $690 \mathrm{~km}$ apogee; the orbital period is $97.1 \mathrm{~min}$. The STEP experiment will be carried out after an initial set-up and calibration phase lasting several weeks. It is expected that some differential acceleration of the test masses will probably be observed, the question is then to decide whether this is due to a violation of the Equivalence Principle or to a disturbance arising from, e.g., helium slosh, gravity gradients, cyclic thermal expansion, residual gas pressure, charging of the test masses, etc.

Such disturbances can be recognised by internal checks, and by repeatedly performing a series of well-defined attitude manoeuvres: placing the spacecraft in a "perpendicular attitude" (rotation by $90^{\circ}$ about the $y$-axis), slowly turning the spacecraft at a rate of $360^{\circ}$ per orbit, and "rotating" the spacecraft about the $z$-axis by an angle $\theta$ (several $\theta$ values will be used). The measurement programme will last six months in total.

\section{Further Development}

After a review of the 22 competing M2 proposals by ESA's scientific advisory bodies in early 1990, STEP was selected together with five other projects for study at the Assessment Phase level as an ESA/ NASA collaborative project. This phase terminated in April 1991 with a presentation to the scientific community. Four missions (see page 209) were then chosen for a Phase A level study, among them STEP. This phase ends in early-1993 and by mid-1993 one of the four will become ESA's M2 mission.

ESA would provide the STEP spacecraft and the European community $50 \%$ of the instrument hardware; NASA would furnish the launcher, operations and the remaining $50 \%$ of the instrument.

\section{SISSA ISAS POST-DOCTORAL POSITIONS Trieste, Italy}

The International School for Advanced Studies (SISSA/ISAS) in Trieste expects to offer a number of post-doctoral positions in the following fields:

Nonlinear analysis

Geometry

Mathematical Physics

Theoretical Particle Physics

Theory of Condensed Matter

Theoretical Astrophysics and Cosmology

Neurophysiology or Molecular Neurobiology

These positions will be available from the Fall of 1992 for one year and renewable for a second year. Candidates should submit their applications by 31 January 1992 with their curriculum vitae, list of published works and their research programme. They should arrange for three letters of reference to be sent by the same date.

Applications and correspondence Postdoc Programme should be sent to:

\author{
International School for Advanced Studies \\ Via Beirut, 2-4 \\ I-34014 Trieste, Italy
}

